\title{
The Comparison of Different Sensory Outputs on the Driving Overtake Alarm System
}

\author{
Yu-Chun Huang, Chia-Jung Tsai, Jo-Yu Kuo, and Fong-Gong Wu \\ Department of Industrial Design, \\ National Cheng Kung University, \\ Tainan 70101, Taiwan \\ \{ging 0215 , sakhing, sc1156\} @gmail.com, \\ fonggong@mail.ncku.edu.tw
}

\begin{abstract}
Most car accidents are caused by improper driving behaviors. Studies have shown that changing lanes improperly is one of the main causes of traffic accidents. This shows that drivers need an assisting alarm system to help them avoid the danger during overtaking. We also found that the existing alarm system and researches try to use different sensory outputs as the alarm signals. However, there were no studies to compare how the different sensory alarm signals affect the drivers. Therefore, in this study, we have setup three kinds of alarm signals (visual, sound, and haptic alarm signals) to see which one is more suitable at the high speed context. On top of that, the sensitivity of the alarm system may be the other key factor that affects drivers' behaviors. So, we manipulate two most commonly shown alarm signal frequencies when the driver feels threatened. The results of this study have proven that the sound and haptic signals are better than visual outputs when drivers are put in a high visual loading situation. This result could be the guideline for future designers of a driving alarm system.
\end{abstract}

Keywords: Overtake, Alarm System, Haptic signal.

\section{Introduction}

With the development of economy, we have more roads and more convenient traffic systems. However, traffic accidents are also increasing. According to the data of Taiwan's Ministry of the Interior that between January and October 2010, the main cause of car accidents is driver negligence (96.07\%). The top three causes of driver negligence are drunk driving (20.1\%), lack of attention (19.5\%), and negligence to traffic rules $(13.8 \%)$. Furthermore, by the report of Taiwan area Freeway Bureau in 2009 , we can see that the first cause of accidents is improper driving, which includes changing lanes improperly and not paying attention to the road, (18.8\%). From the information we understand that car accidents usually happen when drivers want to overtake someone. These factors are due to the fact that drivers could not see the situation of the lane that they are transferring to (sometimes they can not see the following car from the side mirror or rear vision mirror). If there were a system to provide driver alarm signals, it might help to prevent accidents. But, what exact 
components should this alarm contain, and how to present the alarm signals when drivers are in situations of high speed and limited attention? Therefore, in this study we would like to summarize the present researches to understand how to observe and evaluate the participants' driving behaviors and problems by reviewing the existing alarm systems, and try to design a new alarm system to decrease the number of accidents by providing the warnings appropriately.

\section{Driver's Attention and Alarm System}

\subsection{Driver's Attention Allocation}

Human attention could seem as a multi-resource [1], it means that people could arrange their attention to things that are parallel. But, it may be constraint by the physical world. Such as the deployment of central control system, if the switch or button is not arranged by their operational steps or functions, it would add to driver's loading.

Furthermore, driving is a special activity that requires both dividing and focused attention. The impact factors of attention harmonious could be inference from some basic study. For instance, Patrick and Elias [2] made a dual-task performance as it relates to driving, such as tuning a radio or manipulating a cellular phone, drive on the right side of the road: perceptual asymmetries for judgments of automobile proximity. It forces drivers to divide their attention between the traffic demands and the in-car task. The current study examined how mental navigation (spatial distraction) affected accuracy and response time for depth judgments on vehicular stimuli in each visual field. These were compared to a control condition in which no distraction was present, as well as when a semantic (non-spatial) distraction was present two centered tabs, and so on.

\subsection{Relative Issues of Alarm System}

Many alarm systems have already been made and studies. Some studies suggested some principles for alarm system design. For example, Nass et al., analyzed the advantages and disadvantages of the existing systems and propose to focus on signal characteristics, frequency, amplitude, older, compatibility [3]. Some research had pointed out that the modalities of input signals would also interference driver's performance. For instance, some studies find out that compared to visual warning signals, auditory and tactile signals are more effective and have less reaction time at drawing cross modal attention for particular positions, such as rear-end collision situation [4] [5]. Furthermore, some papers indicate that tactile signals were better than auditory and visual [6]. Even talking on the cell phone would shorten the tactile reaction time of drivers [7].

Some studies discussed the location of tactile system in depth. For example, tactile warnings delivered by gas pedal [8] or in the driver's safety restraint or seat [9] [10] [11] is also effective to alert the driver. Atsuo Murata et al. [12] both point out that the driver will respond faster when the tactile signals is shown by the foot vibrator than the steering wheel. 
As for the direction of tactile warning, Cristy Ho et al. [13] indicated that the response was significantly faster with no spatial prediction of vibrating alert. De Vries et al. [14] also showed that the correct rate is not consistent with directional alarm.

From the related work we can know that most of the car accidents is cause by driver negligence. And, the faults of the negligence usaually happens when the drivers try to change lanes. However, this kind of accidents are correalted with the visual attention limitation. Based on the present studies, we can understand that there are many types of alarm systems such as tactile, visual, and auditory. From the existing papers we can arrange them into the following paragraphs.

About the haptic standard, Van Erp has mentioned that they use $250 \mathrm{~Hz}$ vibration in their experiment [11]. On the other hand, Ho and Spence use red and blue LED light for visual warning in their alarm system. Also, many papers discuss the auditory warning system [10] [15] [16]. Most of their auditory warnings are between 75 and $88 \mathrm{~dB}$. And all of them also took environment noise into consideration (about $60 \mathrm{~dB}$ ).

\subsection{Brief Summary}

In this study we would like to summarize the review the existing alarm systems, and try to design a new tactile alarm system to afford drivers different perceptual signals and manipulate the context that when the alarm signals appear. There are two main issues of discussion. Is the driver's attention limitation restricted by the loading of perceptual pathway? We use either visual or tactile signals to see what kinds of perceptual signals would take less effort, and afford driver a useful alarm. The other issue would be the salience effect of the alarm signals. Actually, some of the existing alarm systems present alarm signals when drivers want to change their original way. But, there are still some systems with present alarm signals when the drivers want to change their route "and there are other cars in that route". Therefore, comparing these two situations may prove if the salience effect of the alarm signal interferes with our vigilance.

\section{Method}

\subsection{Participants}

There were 3 male and 3 female graduate school students, age ranging from 25 to 31 (mean=27), served as participants in this experiment. Two strict eligibility requirements to increase the likelihood of all participants having the adequate driving safety knowledge: (a) Have valid driver's license. (b) At least 1 year driving experience within the city.

\subsection{Apparatus}

For the purpose of comparing three kinds of sensory outputs of the overtake alarm system, we have to build up a safety experimental environment for all participants. Therefore, we employed 3DsMAX 2009 software to build up the whole driving environment and render different animations as different treatment levels. There were four segments of the animations and all the films would be taken by the first person view which concluded the front, left and right side of the car. We also use a piece of street sound $(60 \mathrm{~dB})$ as the background noise in all animations. 


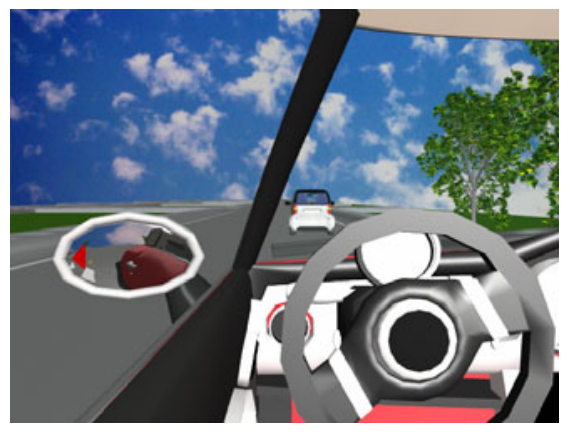

Fig. 1. A sample scene from the driving environment with visual alarm signal

The visual alarm signal was drawn by Photoshop CS3 that was a red triangle with the size of $24 \times 24$ points. The sound alarm signal was a $1000 \mathrm{~Hz}$ pure tone voice with the volume controlled at $80 \mathrm{~dB}$. The haptic alarm signal was made by a motor vibrator that setup on the participants' hands. The location of the haptic signal was decided by our pilot study that the driving wheel was the best location. And, the haptic signal presented was controlled by Arduino. Arduino is an open-source physical computing platform based on a simple microcontroller board. We can write programming script to make the connection with the motor vibrator, and receiving the different trigger as different conditions that are sent from the E-Prime software.

Experiment environment was administered on a projector running at 1280x720 film resolution. Participants control the brake which was made by a keyboard and a pedal by their right foot on the floor.

\subsection{Experimental Design and Procedure}

There were two independent variables we would like to manipulate. First, the alarm signal characteristics (perceptual pathway) either the visual, sound or haptic signals. Second, the frequency (salience effect) of alarm signals: once the other cars would threaten or alarm signals constantly appear. As shown in the figure, the left one indicates that the driver is only threaten while overtaking. However, the right figure shown no matter there were a threat or not, the alarm signals would appear anyway.
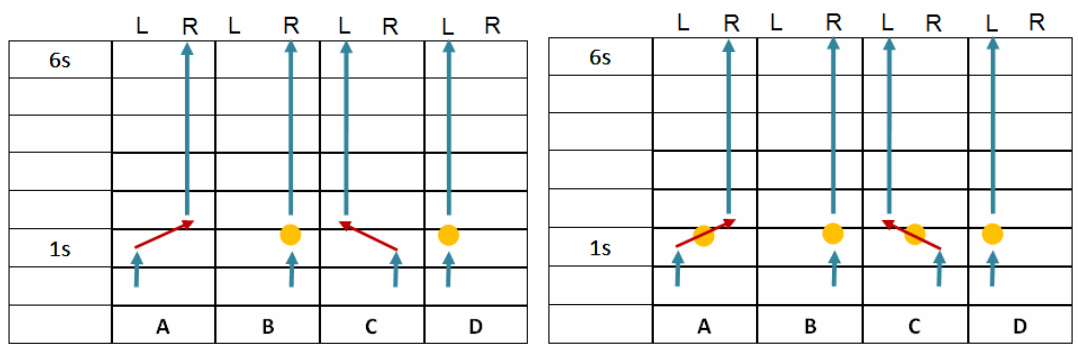

Fig. 2. Eight kinds of possible conditions of experimental design 
A two factors repeated measures of within-participant design was applied as experimental design. Two independent variables were: three kinds of alarm signals (visual, sound, haptic alarm signals); two levels of frequency when the alarm signals appear constantly and when the alarm signals only appear when the driver is under threat. Therefore, the whole experiment contains 96 trials, and it was separated into 6 blocks which contains three kinds of sensory inputs and two kinds of signal frequency. The participants would take different sequences to accomplish the tasks by following the former car under those situations, and they may have to stop the vehicle after each trial. The braking response would be the dependent variable which was the reaction time between the onset of the alarm signal and the braking time.

\section{Result}

As the dependent variable was the reaction time between the onset of the alarm signal and the braking time, we can see the description statistic result as table 1 . In the different sensory outputs, the haptic alarm signals induce the shortest reaction time, and the visual output signals induced the fastest reaction time. In the different levels of the frequency that the alarm signals appear, when the alarm signals constantly appear the participants showed faster reaction times.

Moreover, a two factors repeated measures ANOVA was executed. The result shown on table 2 and figure 5 states that the different alarm signal outputs have main effect $(\mathrm{p}=.047<.05)$. But, the different frequencies of the alarm signals do not show significant difference. However, the non-significant difference comes from the huge difference of the visual outputs.

Table 1. Description statistic

\begin{tabular}{lll}
\hline Variables & Conditions & Reaction Time (MS) \\
\hline Sensory outputs & Visual & 848.79 \\
& Sound & 540.34 \\
& Haptic & 428.54 \\
Frequency & Constantly & 617.06 \\
& Threaten & 617.07 \\
\hline
\end{tabular}

Table 2. Statistic results of repeat measure ANOVA

\begin{tabular}{crrrrr}
\hline Variables & SS & df & MS & \multicolumn{1}{c}{ F } & \multicolumn{1}{c}{ p } \\
\hline Sensory outputs & 591995.10 & 2 & 295997.55 & 5.29 & $.047^{*}$ \\
Output frequency & 5969.58 & 1 & 5969.58 & .28 & .632 \\
Sensory outputs* & 31676.65 & 2 & 15838.33 & .95 & .438 \\
Output frequency & & & & & \\
\hline
\end{tabular}




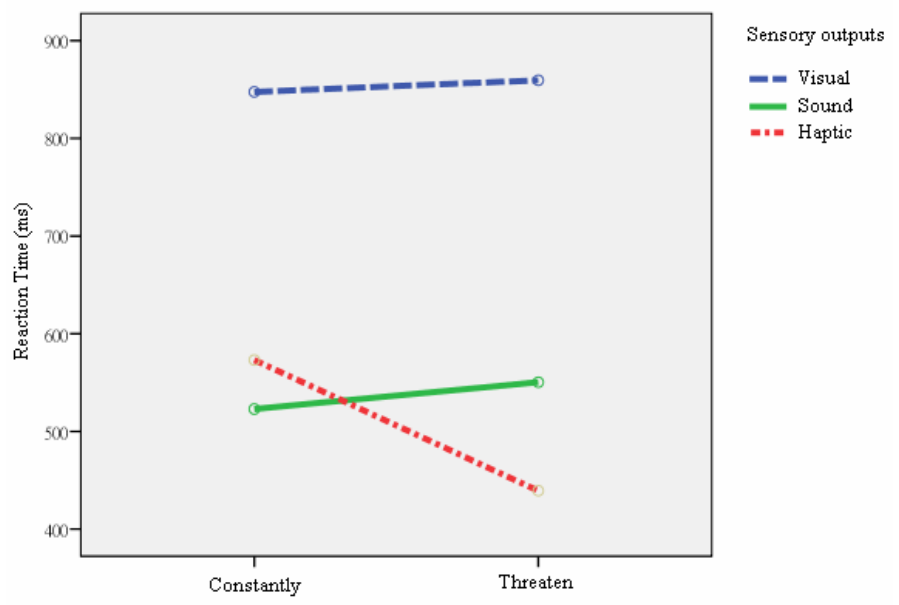

Fig. 3. Reaction time in different conditions

\section{Discussion}

In this study, we used different kinds of the alarm signals which were collected from the existing alarm system and pervious researches. Then, we used three kinds of sensory outputs to compare which one is the most suitable for the overtaking behavior. Therefore, we build up a virtual driving environment and render different animations as our experimental conditions. All participants had to do was to focus on the driving situations and step on the brake when they receive the alarm signals. As the result shown, we can find out how the additional visual alarm signals would interfere with the participants' reaction time when they are under a situation of high visual loadings. Instead, the participants respond more quickly to sound and haptic alarm signals. This result could be corresponding to the Wicken's attentional theory that the human attention is regarded as a multi-resources system [1]. Therefore, when the visual attention is exhausted, we should use other sensory signals as our alarm signal.

However, there are still other extending issues for other sensory outputs, for example the sound and haptic alarm signals do not afford the orientation cues for the participants. And, the strength of the sensory outputs may be interfered by the different driving environment.

The second issue we would like to test was the different frequencies (salience effect) of alarm signals. The signals on the alarm system would either constantly appear or only show when the driver is threatened. We did not find the salience effect. It may be caused by the lower difficulty of the task. Contrast to the real driving situation, in this experiment the participants only have to step on the brake as soon as possible. They did not have to control the gas pedal nor the steering wheel. Also, the experiment was executed trial by trial. Participants may keep the arousal level of alarm signals. Based on those differences of task difficulty and awareness of alarm may immerse the salience effect. 


\section{Conclusion}

In this modern age, the density of personal vehicles is getting higher and higher, also the number of traffic accidents. Studies have shown that changing lanes improperly is one of the main causes of traffic accidents. These factors are due to the fact that drivers could not see the situation of the lane that they are transferring to (sometimes they can not see the following car from the side mirror or rear vision mirror). Some new technologies have emerged to help drivers avoid accidents. However, the engineers who innovate the overtake alarm system did not realize how the different signal outputs could interfere the drivers. So, in this study, we try to compare the different outputs of the alarm system. According to the experiment result, when drivers face high visual loading tasks, the sound and haptic are more suitable than the visual alarm signals. Although the virtual driving environment and the task difficulty could not externalize completely to the real situation, this study still provide some guideline for the driving overtake alarm system.

\section{References}

1. Wickens, C.D.: Processing resources in attention. In: Parasuraman, R., Davies, R. (eds.) Varieties of Attention, pp. 63-101 (1984)

2. Patrick, R.E., Elias, L.J.: Navigational conversation impairs concurrent distance judgments. Accident Analysis and Prevention 41, 36-41 (2009)

3. Nass, C., Jonsson, I., Harris, H., Reaves, B., Endo, J., Brave, S., Takayama, L.: Improving automotive safety by pairing driver emotion and car voice emotion. In: CHI, Portland, Oregon, USA, April 2-7 (2005)

4. Ho, C., Tan, H.Z., Spence, C.: Using spatial vibrotactile cues to direct visual attention in driving scenes. Transportation Research Part F: Traffic Psychology and Behaviour 8, $397-$ $412(2005)$

5. Spence, C., Driver, J.: Cross-modal links in attention between audition, vision, and touch: Implications for interface design. International Journal of Cognitive Ergonomics 1, 351373 (1997)

6. Jeffrey, J., Scott, Rob, G.: Comparison of driver brake reaction times to multimodal rearend collision warnings. In: The Fourth International Driving Symposium on Human Factors in Driver Assessment, Training and Vehicle Design (2009)

7. Mohebbi, R., Gray, R., Tan, H.: Driver Reaction Time to Tactile and Auditory Rear- End Collision Warnings While Talking on a Cell Phone Human Factors. The Journal of the Human Factors and Ergonomics Society 51, 102 (2009)

8. Godthelp, J., Schuman, J.: Intelligent accelerator: An element of driver support. In: Parkes, A.M., Frazen, S. (eds.) Driving Future Vehicles, pp. 265-275. Taylor \& Francis, London (1993)

9. Ho, C., Reed, N., Spence, C.: Assessing the effectiveness of "intuitive" vibrotactile warning signals in preventing frontto- rear-end collisions in a driving simulator. Accident Analysis \& Prevention 38, 989-997 (2006)

10. Ho, C., Spence, C.: Using peripersonal warning signals to orient a driver's gaze. Human Factors 51(4), 539-556 (2009) 
11. Van Erp, J.B.F., van Veen, H.A.H.C.: Vibro-tactile information presentation in automobiles. In: Berber, C., Faint, M., Wall, S., Wing, A.M. (eds.) Proceedings of Eurohaptics 2001, pp. 99-104. University of Birmingham, Birmingham (2001)

12. Atsuo, M., Kohki, T., Makoto, M.: Fifth International Workshop on Computational Intelligence \& Applications (2009)

13. Cristy, H., Hong, Z.T., Charles, S.: Using spatial vibrotactile cues to direct visual attention in driving scenes. Transportation Research Part F 8, 397-412 (2005)

14. De Vries, S.C., Van Erp, J.B.F., Raymond, J.K.: Direction coding using a tactile chair. Applied Ergonomics 40, 477-484 (2008)

15. Mckeown, D., Isherwood, S., Conway, G.: Auditory displays as occasion setters. Hum. Factors 52(1), 54-62 (2010)

16. Rayka, M., Rob, G., Hong, Z.T.: Driver Reaction Time to Tactile and Auditory Rear-End Collision Warnings While Talking on a Cell Phone Human Factors. The Journal of the Human Factors and Ergonomics Society 51, 102 (2009) 